\title{
Small Bowel Adenocarcinoma: Etiology, Presentation, and Molecular Alterations
}

Katrina S. Pedersen, MD, MS ${ }^{a}$; Kanwal Raghav, MBBS, MD' ; and Michael J. Overman, MD $^{\mathrm{b}}$

\begin{abstract}
Small bowel adenocarcinoma (SBA) is a rare cancer that has been treated similarly to colorectal cancer (CRC) in the advanced setting. Incidence has been increasing as detection efforts have been improving for these challenging-to-diagnose tumors, but patients frequently experience prolonged nonspecific symptoms due to delayed diagnosis. As a result of such delays and likely due to variant biology, patient outcomes for SBA are inferior to those for CRC at all stages of diagnosis. Recent molecular studies highlight the genomic differences underpinning these tumors and suggest new future pathways for treatment, distinct from CRC.
\end{abstract}

J Natl Compr Canc Netw 2019;17(9):1135-1141 doi: $10.6004 /$ jnccn.2019.7344

aDivision of Oncology, Washington University School of Medicine, St. Louis, Missouri, and bepartment of Gastrointestinal Medical Oncology, The University of Texas MD Anderson Cancer Center, Houston, Texas.
Although the small intestine constitutes three-quarters of the digestive tract, small bowel adenocarcinoma (SBA) is a rare cancer. As a result, few studies have been published to help guide management of this disease, and resectable and advanced SBA have been primarily treated as an extension of colorectal cancer (CRC). Despite SBA being treated as a large intestinal cancer, patient outcomes are inferior. ${ }^{1}$ In recent years, however, molecular profiling and cooperative efforts have expanded understanding of SBA, and have highlighted settings in which this cancer may not be well-served by being treated as CRC. Consequently, SBA has now received its own set of NCCN Clinical Practice Guidelines in Oncology (NCCN Guidelines), published in this issue. $^{2}$

\section{Epidemiology}

An estimated 10,590 (2.3 per 100,000$)$ patients are expected to be diagnosed with a small intestinal cancer in 2019. ${ }^{3}$ The overall incidence has been increasing slowly since the early 1990s, ${ }^{4,5}$ and historically, adenocarcinoma had been the dominant small intestinal histology, followed by neuroendocrine cancer, lymphoma, and sarcoma (most commonly gastrointestinal stromal tumor and leiomyosarcoma). In approximately $2000,{ }^{6}$ however, neuroendocrine carcinoma became the dominant histology, particularly in distal segments of the small intestine, and now account for $39 \%$ to $45 \%$ of all cases, whereas SBA represents $31 \%$ to $40 \%$ of all small intestinal cancer diagnoses. ${ }^{5-8}$ This means that approximately 3,300 to 4,200 patients in the United States will be diagnosed with SBA this year, comprising only 3\% of total incident digestive cancers. ${ }^{9}$ Most of these primary tumors arise in the duodenum (60\%), with $25 \%$ to $29 \%$ arising in the jejunum and $10 \%$ to $13 \%$ in the ileum ${ }^{10-12}$ (Figure 1). The median age at diagnosis is approximately 60 years, with a higher rate of development seen among men. ${ }^{5,7,11,12}$

Unfortunately, SBA tends to be diagnosed at a later stage compared with CRC. A retrospective comparative outcomes analysis of the SEER-Medicare database found that only $33.7 \%$ of patients with SBA (excluding 

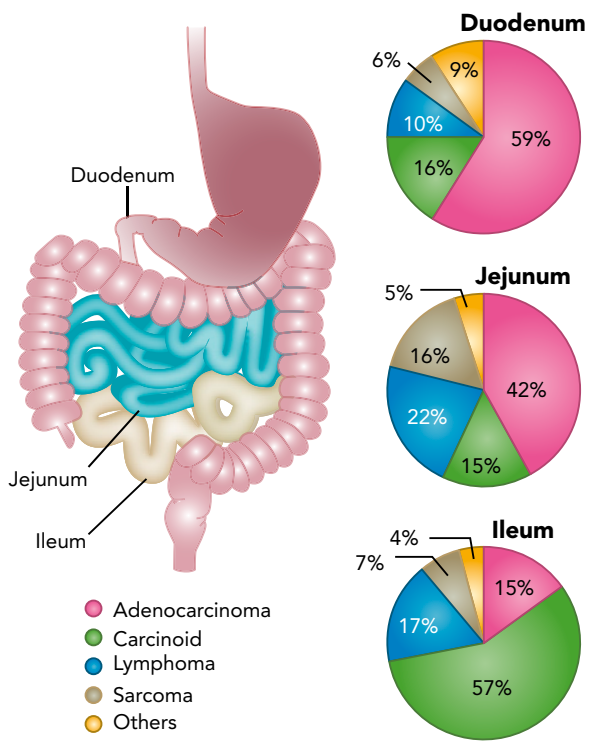

Figure 1. Epidemiology of histologic tumor type stratified by subset of the small bowel from the National Cancer Database (1985-2005), ${ }^{13}$ SEER (1973-2005), ${ }^{10,68}$ and Connecticut Tumor Registry (1980-2000). ${ }^{69}$ Adapted with permission from Springer Nature: Raghav K, Overman MJ. Small bowel adenocarcinomas-existing evidence and evolving paradigms. Nat Rev Clin Oncol 2013;10:534-544.

duodenal) were diagnosed with stage I-II disease compared with $52.3 \%$ of those with colon cancer, ${ }^{1}$ and $32.1 \%$ of patients with SBA were initially diagnosed with distant metastases compared with $15.6 \%$ of those with CRC. Findings from a National Cancer Database (NCDB) analysis of patients with SBA from 2004 through 2013 align with those from the SEER-Medicare study. ${ }^{13}$ Taken together, these studies suggest the difficulty in detecting these cancers ${ }^{1}$ and highlight the lack of screening programs, even for potentially high-risk individuals.

\section{Etiology}

The reason for SBA's rarity remains unclear. There are multiple theories, ${ }^{14}$ including the relatively dilute contents of digestive material passing through the small intestine causing less irritation to the mucosa compared with the lower water content of feces within the large intestine. This theory could partially explain why twothirds of adenocarcinomas arise in the duodenum, where the mucosa encounters the more caustic $\mathrm{pH}$ of gastric chyme as well as irritation from bile and active pancreatic enzymes once past the ampulla. Additionally, the small intestine does not have a "warehouse" function that keeps potential harmful ingested elements in proximity to tissue for hours at a time, unlike the large intestine's significantly slower transport of stool in normal circumstances. ${ }^{7}$ The small intestine has been shown to have a different redox microenvironment from that found in the colon. ${ }^{15}$ Specifically, fewer reactive oxygen species are present or generated within the small intestine compared with the colon. During times of oxidative stress, DNA adducts form more readily within the colon than the small bowel, suggesting greater likelihood of DNA damage and therefore higher rates of colorectal tumor transformation. ${ }^{15}$ The small intestine is a major site of immunosurveillance in the body. Dendritic cells present within the mucosa have been found to sample antigens from food, microbiota, and blood that subsequently modulate activity of CD4+ and CD8+ lymphocytes. ${ }^{16}$ Furthermore, increased lymphoid tissue density throughout the small bowel will release more IL-10 compared with other sites in the body, which has been found to be protective against cell transformation. ${ }^{17}$ It remains unclear what role the gut microbiota play in promoting or preventing small bowel carcinogenesis, and is another area of research opportunity.

Some SBA risk factors have been identified, primarily in retrospective and registry analyses. Among environmental exposures, alcohol and cigarette smoking may lead to increased risk of SBA carcinogenesis. ${ }^{18,19}$ An exploratory analysis of occupations with the highest calculated odds of developing SBA included building caretakers, housekeepers, farm laborers, dock workers, dry cleaners, textile workers, and welders. ${ }^{20} \mathrm{~A}$ number of dietary associations have been investigated, with findings showing that refined carbohydrates, red meats, saturated fat, cured meats, smoked foods may increase risk of SBA, whereas fruit, vegetables, coffee, dietary fiber, and fish may be protective. ${ }^{18,21-25}$ However, the studies have been small, sometimes conflicting, and subject to potential recall bias.

Several hereditary cancer syndromes can predispose individuals to developing SBA. Hereditary nonpolyposis CRC (HNPCC) or Lynch syndrome is an autosomal dominant inheritance of germline mutations in DNA mismatch repair (MMR) genes, including $M L H 1, M S H 2$, MSH6, and PMS2, and rarely EPCAM and PMS1. These mutations predispose family members not only to SBA and CRC but also to endometrial, ovarian, gastric, biliary, upper urinary tract, and skin cancers. The lifetime risk of developing SBA in Lynch-affected individuals remains low at about $1 \%$, according to European registry studies, ${ }^{26,27}$ and therefore no small bowel screening recommendations currently exist. Much like colon cancer, however, HNPCC is responsible for approximately $5 \%$ to $10 \%$ of cancers. ${ }^{28,29}$ Thus, routinely assessing all SBA tumors for deficient MMR (dMMR) gene expression or high microsatellite instability (MSI-H) is indicated and may help predict better therapies, including immune checkpoint therapy, for these patients. ${ }^{30,31}$ Familial adenomatous polyposis (FAP) has been typically associated with the formation of 
hundreds of colon polyps and early-onset CRC. These adenomatous polyps, which form readily due to germline inactivation of the $A P C$ gene, can also occur within the small intestine. $A P C$ is a member of the $\mathrm{Wnt} / \beta$-catenin signaling pathway, which is frequently implicated in CRC. Individuals affected by FAP have an approximately $4.5 \%$ chance of developing SBA, which is the most common cancer-related cause of death in individuals who have had total proctocolectomy. ${ }^{32,33}$ Finally, PeutzJeghers syndrome, in inherited mutation of STK11, can also cause polyposis throughout the small intestine. As a result, affected individuals may have a relative risk of 520 for developing SBA compared with unaffected persons. ${ }^{34}$

Proinflammatory conditions may also contribute to the development of SBA. Among these, celiac disease and Crohn's disease are the best characterized. In registry studies and case series, $0.7 \%$ to $13 \%$ of patients with celiac disease were found to have small bowel adenoma or SBA, ${ }^{35-37}$ which translates to a 34-fold increased risk of developing SBA. ${ }^{38}$ In general, these patients may be younger at disease onset and potentially demonstrate better overall survival than those with SBA caused by other disorders. ${ }^{37}$ Unlike most other causes of SBA, Crohn's disease tends to present with cancers in the distal small bowel, primarily the ileum. Patients diagnosed with Crohn's disease have a 21 - to 60 -fold increased risk of developing $\mathrm{SBA},{ }^{39,40}$ which appears to be associated with duration of Crohn's diagnosis, with median presentation of adenocarcinoma approximately 8 years after diagnosis of inflammatory bowel disease. ${ }^{39}$

\section{Clinical Presentation}

Unlike CRC, SBA has no population-based screening, largely because its rarity creates a low value for screening among the general population. This also reflects the lack of tools to noninvasively and reliably detect tumors in a cost-effective manner. As a result, this disease tends to present at later stages and, due to the nonspecificity of symptoms, have significant delays to diagnosis, ranging on average between 7 and 8 months from onset of symptoms. ${ }^{41,42}$

Among patients diagnosed with SBA, intermittent abdominal pain is the most frequent complaint. ${ }^{41,43}$ Fewer than half of patients will present with accompanying weight loss, nausea, or vomiting, and only about a third will experience any gastrointestinal bleeding, which may present as iron deficiency anemia with negative findings on colonoscopy or even standard esophagogastroduodenoscopy (EGD). Due to the difficulty and delay in diagnosing, patients may present with intestinal obstruction $(22 \%-26 \%)$ and less commonly with bowel perforation $(6 \%-9 \%)$. As a result of these delays, it is not surprising that patients with SBA present with more advanced disease compared with those with CRC, highlighting the importance of performing further tests in patients with these persistent symptoms.

\section{Diagnostic Workup}

SBAs have been traditionally difficult to diagnose due to most of the small intestine's length being inaccessible to traditional scoping techniques and due to the difficulty of capturing intraluminal masses radiographically until they become more advanced. Several methods can enhance the amount of intestinal surface area that can be visualized beyond EGD or colonoscopy with terminal ileal examination, including push enteroscopy, video capsule endoscopy (VCE), and deep small bowel enteroscopy (ie, balloon enteroscopy and spiral enteroscopy).

Radiographic imaging may detect SBA, although CT and MRI may only detect these malignancies in cases of small bowel obstruction. CT enteroclysis, which is performed with nasogastric infusion of contrast to the small intestine, has improved sensitivity of up to $93 \%$ and specificity of $99 \%$ in a meta-analysis ${ }^{44}$ and may have better sensitivity than MR enteroclysis. ${ }^{45}$ Cross-sectional enterography (CT enterography [CTE] and MR enterography [MRE]), wherein patients drink the contrast volume, has now largely supplanted the more-invasive enteroclysis techniques, with tolerable sensitivity (76\% CTE, 93\% MRE) and specificity (95\% CTE, 99\% MRE) in detecting small bowel lesions. ${ }^{46}$

VCE is in widespread use and has been reported to detect small intestinal tumors in $4 \%$ to $9 \%$ of patients with occult gastrointestinal bleed. ${ }^{47,48}$ Unfortunately, its utility is limited by the inability to obtain biopsy or intervene therapeutically (eg, if a tumor is bleeding). Its use is contraindicated in patients presenting with bowel obstruction, which can cause capsule retention. An Australian series of small bowel tumors found that, of 26 patients with tumors, only $35 \%$ were detected by radiology prior to VCE, and 5 had SBA that underwent resection..$^{49}$ Only 4 of these 5 patients remained diseasefree, highlighting the poor outcomes of SBA.

Push enteroscopy uses scopes longer than standard EGD equipment and a rigid overtube to allow deeper penetration into the small intestine. However, some studies have shown that this technique missed lesions in $26 \%$ of patients with occult gastrointestinal bleeding. ${ }^{50}$ When compared with VCE, which missed only $8 \%$ of lesions, it may have inferior yield, although it provides greater potential for therapeutic intervention to control bleeding during the procedure.

Double-balloon enteroscopy (DBE) allows the endoscope to reach the anterograde depth of 220 to $360 \mathrm{~cm} .{ }^{51}$ 
Despite this enhanced depth of examination, metaanalysis comparing VCE and DBE found similar outcomes between the procedures, with both identifying underlying tumors in patients with occult gastrointestinal bleeding in $11 \%$ of cases. ${ }^{52}$ Few studies report on the sensitivity/specificity of DBE or spiral enteroscopy in detecting SBA.

Unfortunately, there are few prospective, randomized trials assessing which direct visualization modality is superior in diagnosing SBA. Thus, the procedure should be determined at the discretion of the gastroenterologist based on exhibited symptoms, patient characteristics, and location of the area to be examined.

\section{Molecular Alterations}

Recent studies have made major strides in understanding the molecular drivers of SBA. These efforts have demonstrated SBA to represent a unique molecular entity with distinct differences between both CRC and gastric cancer.

One recent study has provided tremendous insights through a comparative analysis of molecular alterations detected from either a 236 or 315 cancer-related gene panel between SBA $(n=317)$, CRC $(n=6,353)$, and gastric adenocarcinoma $(n=889)$ (Figure 2$).{ }^{53}$ Of the top 20 most commonly altered genes, 12 were statistically different between SBA and CRC, with the most notable differences in $A P C$ (26.8\% vs $75.9 \%$; $P<.001)$, TP53 (58.4\% vs $75 \%$; $P<.001)$, and $C D K N 2 A(14.5 \%$ vs $2.6 \% ; P<.001)$, respectively. For the comparison of SBA and gastric cancer, 12 genes were statistically different, with the most notable being KRAS (53.6\% vs $14.2 \%$; $P<.001), A P C(26.8 \%$ vs $7.8 \% ; P<.001$ ), and $S M A D 4$ (17.4\% vs $5.2 \% ; P<.001$ ). The low rate of $A P C$ alterations in SBA compared with CRC has been noted in prior smaller studies and represents one of the most fundamental genomic differences between large bowel adenocarcinoma and SBA. ${ }^{54-57}$ In another comparative analysis of copy number changes between SBA, CRC, and gastric cancer, unsupervised clustering showed that SBA clustered more commonly with CRC than gastric cancer. ${ }^{58}$

Another critical molecular insight relates to the comparison of molecular alterations across the different small bowel subsites: duodenum, jejunum, and ileum. In general, these efforts have demonstrated similar molecular alteration rates across the small bowel. ${ }^{53}$ This finding supports the view that anatomic differences represent the main driver for the worse patient outcomes observed when comparing duodenal versus jejunal/ileal adenocarcinomas. ${ }^{59}$ No statistical differences were seen among the top 5 most commonly altered genes (TP53, KRAS, APC, SMAD4, and PIK3CA) when comparing duodenal adenocarcinoma versus cancers of other small bowel sites from 317 SBA cases. ${ }^{53}$ However, both $C D K N 2 A$ $(18 \%$ vs $10 \% ; P<.05)$ and $E R B B 2(13 \%$ vs $4 \% ; P<.01)$ alterations were noted to be higher in duodenal adenocarcinomas. This finding was also confirmed in a study of 83 patients, in which ERBB2 mutations were noted to be more common in duodenal versus jejunal/ileal adenocarcinomas ( $15.8 \%$ vs $2.2 \%$; $P=.04){ }^{60}$

In addition to the pattern of genomic alterations, unique differences in SBA compared with neighboring cancers exist with regard to the type of molecular alterations. Although in CRC most BRAF alterations are

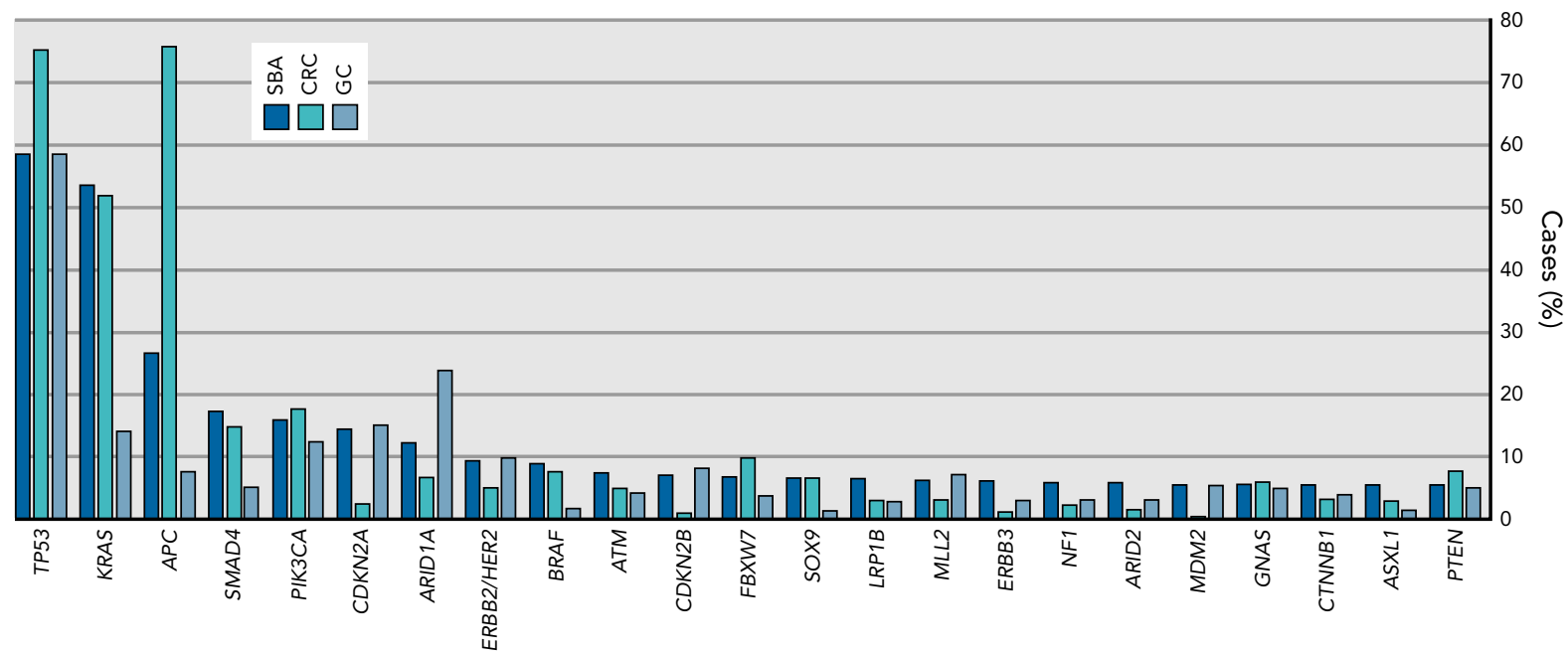

Figure 2. Genes altered in $>5 \%$ of small bowel adenocarcinoma (SBA) compared with colorectal cancer (CRC) and gastric carcinoma (GC) cases from a total of 7,559 patients who underwent genomic sequencing on a 236 or 315 cancer-related gene panel.

Adapted from Schrock AB, Devoe CE, McWilliams R, et al. Genomic profiling of small-bowel adenocarcinoma. JAMA Oncol 2017;3:1546-1553. Reproduced with permission from the American Medical Association. (02017. All right reserved. 
$B R A F$ V600E, this codon is infrequently altered in SBA. For example, in the previously mentioned analysis of 317 SBA cases, BRAF alterations occurred in similar rates between SBA and CRC (9.1\% vs $7.6 \%$, respectively). However, only $10.3 \%$ of $B R A F$ alterations were $B R A F$ V600E for SBA compared with $73.2 \%$ for CRC. ${ }^{53}$ A similar finding was found in an analysis of 106 cases in which 11 (10.4\%) patients had BRAF mutations but none were $B R A F$ V600E. ${ }^{61}$ Distinct differences also exist for ERBB2. Despite a similar rate of $E R B B 2$ alterations between SBA and gastric cancer (9.5\% for both), the rate of amplification differs greatly, with only $23 \%$ of alterations being amplification in SBA compared with $69 \%$ in gastric cancer. Although the rate of $E R B B 2$ alterations is lower in CRC (5.1\%), most alterations are amplifications (66\%). This finding is similar to 3 other studies, which report $70 \%$ to $76 \%$ of the ERBB2 genomic alterations were mutations. ${ }^{60-62}$ Studies have reported high rates of MSI-H or dMMR in SBA, with a rate of $21.6 \%$ in an 83 -patient series ${ }^{60}$ and $14.2 \%$ in a 106 -patient series. ${ }^{61}$ However, these rates are highly influenced by the stage distribution of the population, with higher rates seen in earlier-stage disease. In the previously mentioned comparison study of primarily advanced-stage patients, a slightly higher rate of SBA (7.6\%) was seen when compared with CRC and gastric cancer (4\% and 3.9\%, respectively)..$^{53}$ The etiology for MSI-H appears to be more commonly related to Lynch syndrome than MLH-1 methylation in contrast to CRC, but outcomes for patients with earlier-stage disease are improved in CRC and SBA. ${ }^{63}$ For patients with microsatellite-stable tumors, the tumor mutational burden appears low, with 2 studies reporting median burdens of $4.2^{53}$ and $4.3^{61}$ mutations per megabase, respectively.

Although most SBAs are sporadic in nature, inflammatory bowel disease, particular Crohn's disease, celiac disease, and Lynch syndrome, are predisposing conditions for a small subset of patients. Although the small number of patients within these subsets have limited molecular comparisons, studies of celiac-associated SBA have demonstrated consistently high rates of MSI-H, ranging from $50 \%$ to $73 \% .{ }^{61,64,65}$ Interestingly, in one study of 15 celiac disease_related SBA, all 10 of the MSI-H cases demonstrated MLH1 methylation, suggesting a role of hypermethylation as a causative factor in celiac-related SBA. ${ }^{64}$

A number of genomic alterations exist between CRC and SBA that may have direct therapeutic implications. Although ERBB2 alterations are present in SBA, they are primarily point mutations, unlike CRC, and preclinical data has suggested that $E R B B 2$ tyrosine kinase inhibitors may have a role in the treatment of SBA. ${ }^{62}$ In addition, the role of anti-epidermal growth factor receptor (EGFR) therapy in $R A S$ wild-type SBA is unclear, with a small clinical trial of single agent anti-EGFR therapy with panitumumab showing no responses in 9 patients with SBA. ${ }^{66}$ This outcome may reflect the midgut derivation for the small intestine, which represents one of the major hypotheses for the differential benefit of anti-EGFR therapy in left-sided versus right-sided CRC. Thus, based on current data, the most promising potential clinical targets in SBA are dMMR, ERBB2-activating mutations, and atypical $B R A F$ mutations.

Comparison With Large Bowel Adenocarcinoma Historically, management of SBA has been based on the treatment approach for large bowel adenocarcinoma or CRC. However, a number of distinct differencesmost notably anatomic and molecular-have emerged that strongly support the need to better tailor the clinical management of SBA. These differences underscore the importance of guidelines to enable optimal care delivery and delineate areas for essential research efforts. To meet this need, NCCN has now published guidelines for the treatment of SBA, available in this issue. $^{2}$

One of the most dramatic differences between these cancers relates to the marked difference in incidence. In fact, while CRC is declining in incidence, SBA is increasing. ${ }^{67}$ The lack of cancer screening approaches, lower incidence, and challenge of luminal visualization all contribute to delayed diagnosis. These factors may explain the higher rate of stage IV presentation for SBA compared with colon cancer (32\% vs $20 \%) .{ }^{67}$ In a large population-based comparison between these 2 cancers using the SEER database (SBA, $n=4,518$ vs large bowel adenocarcinoma, $n=261,521$ ), the stage-by-stage outcome was significantly worse for patients with SBA compared with large bowel adenocarcinoma. ${ }^{67}$ Even with normalization for lymph node assessment, cancerspecific survival among patients with SBA remained consistently lower by approximately $10 \%$ to $15 \%$ for each stage of disease (stage I, II and III). For example, stage I jejunal/ileal adenocarcinomas with $\geq 8$ lymph nodes removed demonstrated a cancer-specific survival of $81.6 \%$ compared with $93.3 \%$ for colon cancer. Such a finding appears to support an underlying difference in biology between these cancers.

The duodenum represents a unique anatomic site within the small bowel due to the retroperitoneal nature of the second and third portions and the anatomic complexity of pancreatic and biliary drainage into the site. As such, a pancreaticoduodenectomy is often required for surgical resection and there is a greater locoregional risk of recurrence. In part, the worse outcomes for duodenal compared with jejunal/ ileal adenocarcinomas reflect this more complex anatomy. Management of duodenal adenocarcinomas 
thus requires a unique approach compared with colon cancer.

\section{Conclusions}

Although extrapolations from CRC have helped guide therapeutic decisions for SBA, recent refinement of the understanding of this rare cancer warranted the establishment of specific guidelines that address the unique nature of this cancer. In particular, the symptom presentation, diagnostic workup, predisposing conditions, surgical approach, and use of targeted therapy represent distinct and relevant clinical differences from CRC. Recently, an improved molecular understanding of SBA has also demonstrated this cancer to be unique from its neighboring intestinal cancers and strongly supports the need for further research efforts to better understand this rare cancer. As a result of these new insights, NCCN has now published guidelines for the treatment of SBA, available in this issue. ${ }^{2}$

Submitted June 6, 2019; accepted for publication August 6, 2019

Disclosures: Dr. Pedersen has disclosed that she receives grant/research support from AbbVie and Merck, and consulting fees/honoraria from Array and BeiGene. The remaining authors have disclosed that they have no financial interests, arrangements, affiliations, or commercial interests with the manufacturers of any products discussed in this article or their competitors.

Correspondence: Katrina S. Pedersen, MD, MS, Division of Oncology, Washington University School of Medicine, 660 South Euclid Avenue, Campus Box 8056, St. Louis, MO 63110. Email: kpedersen@wustl.edu

\section{References}

1. Young Jl, Mongoue-Tchokote S, Wieghard N, et al. Treatment and survival of small-bowel adenocarcinoma in the United States: a comparison with colon cancer. Dis Colon Rectum 2016;59:306-315.

2. Benson AB III, Venook AP, Al-Hawary MM, et al. NCCN Clinical Practice Guidelines in Oncology: Small Bowel Adenocarcinoma. Version 1.2020. To view the most recent version, visit NCCN.org. Accessed August 7, 2019

3. National Cancer Institute. Cancer stat facts: small intestine cancer, 2019 Surveillance, Epidemilogy, and End Results Program. Available at: https://seer.cancer.gov/statfacts/html/smint.html. Accessed August 7, 2019

4. Severson RK, Schenk M, Gurney JG, et al. Increasing incidence of adenocarcinomas and carcinoid tumors of the small intestine in adults. Cancer Epidemiol Biomarkers Prev 1996;5:81-84

5. Bilimoria KY, Bentrem DJ, Wayne JD, et al. Small bowel cancer in the United States: changes in epidemiology, treatment, and survival over the last 20 years. Ann Surg 2009;249:63-71.

6. Pan SY, Morrison H. Epidemiology of cancer of the small intestine. World J Gastrointest Oncol 2011;3:33-42.

7. Aparicio T, Zaanan A, Svrcek M, et al. Small bowel adenocarcinoma: epidemiology, risk factors, diagnosis and treatment. Dig Liver Dis 2014; 46:97-104.

8. Legué LM, Bernards N, Gerritse SL, et al. Trends in incidence, treatment and survival of small bowel adenocarcinomas between 1999 and 2013: a population-based study in The Netherlands. Acta Oncol 2016;55: 1183-1189.

9. Jemal A, Siegel R, Ward E, et al. Cancer statistics, 2009. CA Cancer J Clin 2009:59:225-249.

10. Qubaiah O, Devesa SS, Platz CE, et al. Small intestinal cancer: a population-based study of incidence and survival patterns in the United States, 1992 to 2006. Cancer Epidemiol Biomarkers Prev 2010;19: 1908-1918.

11. Dabaja BS, Suki D, Pro B, et al. Adenocarcinoma of the small bowel: presentation, prognostic factors, and outcome of 217 patients. Cancer 2004; 101:518-526.

12. Halfdanarson TR, McWilliams RR, Donohue JH, Quevedo JF. A singleinstitution experience with 491 cases of small bowel adenocarcinoma. Am J Surg 2010;199:797-803.

13. Mehmet $\mathrm{A}$, Jiang $\mathrm{M}, \mathrm{Wu} \mathrm{C}$, et al. Incidence, treatment and survival outcomes of small bowel adenocarcinomas: a National Cancer Database (NCDB) analysis [abstract]. J Clin Oncol 2018;36(Suppl): Abstract e16262.

14. Schottenfeld D, Beebe-Dimmer JL, Vigneau FD. The epidemiology and pathogenesis of neoplasia in the small intestine. Ann Epidemiol 2009;19: 58-69.

15. Sanders LM, Henderson CE, Hong MY, et al. Pro-oxidant environment of the colon compared to the small intestine may contribute to greater cancer susceptibility. Cancer Lett 2004;208:155-161.

16. Chang SY, Song JH, Guleng B, et al. Circulatory antigen processing by mucosal dendritic cells controls CD8(+) T cell activation. Immunity 2013; 38:153-165.
17. Dennis KL, Saadalla A, Blatner NR, et al. T-cell expression of IL10 is essential for tumor immune surveillance in the small intestine. Cancer Immunol Res 2015;3:806-814

18. Wu AH, Yu MC, Mack TM. Smoking, alcohol use, dietary factors and risk of small intestinal adenocarcinoma. Int J Cancer 1997;70:512-517.

19. Kaerlev L, Teglbjaerg PS, Sabroe S, et al. Is there an association between alcohol intake or smoking and small bowel adenocarcinoma? Results from a European multi-center case-control study. Cancer Causes Control 2000, 11:791-797.

20. Kaerlev L, Teglbjaerg PS, Sabroe S, et al. Occupation and small bowe adenocarcinoma: a European case-control study. Occup Environ Med 2000;57:760-766.

21. Chow WH, Linet MS, McLaughlin JK, et al. Risk factors for small intestine cancer. Cancer Causes Control 1993;4:163-169.

22. Negri E, Bosetti C, La Vecchia C, et al. Risk factors for adenocarcinoma of the small intestine. Int J Cancer 1999;82:171-174.

23. Neugut Al, Jacobson JS, Suh S, et al. The epidemiology of cancer of the small bowel. Cancer Epidemiol Biomarkers Prev 1998;7:243-251.

24. Cross AJ, Leitzmann MF, Subar AF, et al. A prospective study of meat and fat intake in relation to small intestinal cancer. Cancer Res 2008;68: 9274-9279.

25. Schatzkin A, Park Y, Leitzmann MF, et al. Prospective study of dietary fiber whole grain foods, and small intestinal cancer. Gastroenterology 2008; 135:1163-1167.

26. Aarnio M, Mecklin JP, Aaltonen LA, et al. Life-time risk of different cancers in hereditary non-polyposis colorectal cancer (HNPCC) syndrome. Int J Cancer 1995;64:430-433

27. Bonadona $\mathrm{V}$, Bonaïti $\mathrm{B}$, Olschwang $\mathrm{S}$, et al. Cancer risks associated with germline mutations in MLH1, MSH2, and MSH6 genes in Lynch syndrome. JAMA 2011:305:2304-2310.

28. Rodriguez-Bigas MA, Vasen HF, Lynch HT, et al. Characteristics of small bowel carcinoma in hereditary nonpolyposis colorectal carcinoma. International Collaborative Group on HNPCC. Cancer 1998;83:240-244

29. Zhang MQ, Chen ZM, Wang HL. Immunohistochemical investigation of tumorigenic pathways in small intestinal adenocarcinoma: a comparison with colorectal adenocarcinoma. Mod Pathol 2006;19:573-580.

30. Schulmann K, Brasch FE, Kunstmann E, et al. HNPCC-associated small bowel cancer: clinical and molecular characteristics. Gastroenterology 2005; 128:590-599.

31. Le DT, Uram JN, Wang H, et al. PD-1 blockade in tumors with mismatchrepair deficiency. N Engl J Med 2015;372:2509-2520.

32. Jagelman DG, DeCosse JJ, Bussey HJ. Upper gastrointestinal cancer in familial adenomatous polyposis. Lancet 1988;1:1149-1151.

33. Bülow $\mathrm{S}$, Alm T, Fausa $O$, et al., Duodenal adenomatosis in familial adenomatous polyposis. DAF Project Group. Int J Colorectal Dis 1995;10: 43-46.

34. Giardiello FM, Brensinger JD, Tersmette AC, et al. Very high risk of cancer in familial Peutz-Jeghers syndrome. Gastroenterology 2000;119: 1447-1453. 
35. Rampertab SD, Forde KA, Green PH. Small bowel neoplasia in coeliac disease. Gut 2003;52:1211-1214.

36. Howdle PD, Holmes GK. Small bowel malignancy in coeliac disease. Gut 2004;53:470.

37. Caio $G$, Volta $U$, Ursini $F$, et al. Small bowel adenocarcinoma as a complication of celiac disease: clinical and diagnostic features. BMC Gastroenterol 2019;19:45.

38. Green PH, Fleischauer AT, Bhagat $G$, et al. Risk of malignancy in patients with celiac disease. Am J Med 2003;115:191-195.

39. von Roon AC, Reese G, Teare J, et al. The risk of cancer in patients with Crohn's disease. Dis Colon Rectum 2007;50:839-855

40. Cahill C, Gordon PH, Petrucci A, Boutros M. Small bowel adenocarcinoma and Crohn's disease: any further ahead than 50 years ago? World J Gastroenterol 2014;20:11486-11495.

41. Ciresi DL, Scholten DJ. The continuing clinical dilemma of primary tumors of the small intestine. Am Surg 1995;61:698-702; discussion 702-703.

42. Maglinte DD, O'Connor K, Bessette J, et al. The role of the physician in the late diagnosis of primary malignant tumors of the small intestine. Am J Gastroenterol 1991;86:304-308.

43. Talamonti MS, Goetz LH, Rao S, Joehl RJ. Primary cancers of the small bowel: analysis of prognostic factors and results of surgical management. Arch Surg 2002;137:564-570; discussion 570-571.

44. Soyer P, Aout M, Hoeffel C, et al. Helical CT-enteroclysis in the detection of small-bowel tumours: a meta-analysis. Eur Radiol 2013;23:388-399.

45. Schmidt S, Lepori D, Meuwly JY, et al. Prospective comparison of MR enteroclysis with multidetector spiral-CT enteroclysis: interobserver agreement and sensitivity by means of "sign-by-sign" correlation. Eur Radiol 2003;13:1303-1311.

46. Masselli G, Di Tola M, Casciani E, et al. Diagnosis of small-bowel diseases: prospective comparison of multi-detector row CT enterography with MR enterography. Radiology 2016;279:420-431.

47. Cheung DY, Kim JS, Shim KN, et al. The usefulness of capsule endoscopy for small bowel tumors. Clin Endosc 2016;49:21-25.

48. Cobrin GM, Pittman RH, Lewis BS. Increased diagnostic yield of small bowel tumors with capsule endoscopy. Cancer 2006;107:22-27.

49. Bailey AA, Debinski HS, Appleyard MN, et al. Diagnosis and outcome of small bowel tumors found by capsule endoscopy: a three-center Australian experience. Am J Gastroenterol 2006;101:2237-2243.

50. de Leusse A, Vahedi K, Edery J, et al. Capsule endoscopy or push enteroscopy for first-line exploration of obscure gastrointestinal bleeding? Gastroenterology 2007;132:855-862; quiz 1164-1165.

51. Di Caro S, May A, Heine DG, et al. The European experience with doubleballoon enteroscopy: indications, methodology, safety, and clinical impact. Gastrointest Endosc 2005;62:545-550.

52. Pasha SF, Leighton JA, Das A, et al. Double-balloon enteroscopy and capsule endoscopy have comparable diagnostic yield in small-bowel disease: a meta-analysis. Clin Gastroenterol Hepatol 2008;6:671-676.
53. Schrock AB, Devoe CE, McWilliams $R$, et al. Genomic profiling of smallbowel adenocarcinoma. JAMA Oncol 2017;3:1546-1553.

54. Lowenfels $A B$. Why are small-bowel tumours so rare? Lancet 1973;1: 24-26.

55. Calman KC. Why are small bowel tumours rare? An experimental model. Gut 1974;15:552-554.

56. Bläker $\mathrm{H}$, Helmchen $\mathrm{B}$, Bönisch $\mathrm{A}$, et al. Mutational activation of the RAS RAF-MAPK and the Wnt pathway in small intestinal adenocarcinomas. Scand J Gastroenterol 2004;39:748-753.

57. Alvi MA, McArt DG, Kelly P, et al. Comprehensive molecular pathology analysis of small bowel adenocarcinoma reveals novel targets with potential for clinical utility. Oncotarget 2015;6:20863-20874.

58. Haan JC, Buffart TE, Eijk PP, et al. Small bowel adenocarcinoma copy number profiles are more closely related to colorectal than to gastric cancers. Ann Oncol 2012;23:367-374.

59. Overman MJ, Hu CY, Wolff RA, Chang GJ. Prognostic value of lymph node evaluation in small bowel adenocarcinoma: analysis of the Surveillance, Epidemiology, and End Results database. Cancer 2010;116:5374-5382.

60. Laforest A, Aparicio T, Zaanan A, et al. ERBB2 gene as a potential therapeutic target in small bowel adenocarcinoma. Eur J Cancer 2014;50: 1740-1476.

61. Hänninen UA, Katainen R, Tanskanen T, et al., Exome-wide somatic mutation characterization of small bowel adenocarcinoma. PLoS Genet 2018; 14:e1007200.

62. Adam L, San Lucas FA, Fowler R, et al. DNA sequencing of small bowe adenocarcinomas identifies targetable recurrent mutations in the ERBB2 signaling pathway. Clin Cancer Res 2019;25:641-651.

63. Aparicio T, Svrcek M, Zaanan A, et al., Small bowel adenocarcinoma phenotyping, a clinicobiological prognostic study. Br J Cancer 2013;109: 3057-3066.

64. Diosdado B, Buffart TE, Watkins R, et al. High-resolution array comparative genomic hybridization in sporadic and celiac disease-related smal bowel adenocarcinomas. Clin Cancer Res 2010;16:1391-1401.

65. Potter DD, Murray JA, Donohue JH, et al. The role of defective mismatch repair in small bowel adenocarcinoma in celiac disease. Cancer Res 2004 64:7073-7077.

66. Gulhati P, Raghav K, Shroff R, et al. Phase II study of panitumumab in RAS wild-type metastatic adenocarcinoma of small bowel or ampulla of vater. Oncologist 2018;23:277-e26.

67. Overman MJ, Hu CY, Kopetz S, et al. A population-based comparison of adenocarcinoma of the large and small intestine: insights into a rare disease. Ann Surg Oncol 2012;19:1439-1445.

68. Chow JS, Chen CC, Ahsan H, Neugut Al. A population-based study of the incidence of malignant small bowel tumours: SEER, 1973-1990. Int J Epidemiol 1996;25:722-728.

69. Hatzaras I, Palesty JA, Abir F, et al. Small-bowel tumors: epidemiologic and clinical characteristics of 1260 cases from the connecticut tumor registry. Arch Surg 2007;142:229-235 